\section{Cost-effectiveness of population genomic screening}

To the Editor:

Zhang and colleagues recently published an analysis of the potential economic value of population genomic screening of all young adults in a health-care system. ${ }^{1}$ The analysis considered disease risk screening for hereditary breast and ovarian cancer (HBOC) and Lynch syndrome, and carrier screening for cystic fibrosis, spinal muscular atrophy, and fragile X syndrome. The authors found screening would lead to health-care cost savings and prevention of disability-adjusted life-years (DALYs) and would be highly cost-effective with an incremental cost-effectiveness ratio of AUD\$4038/DALY prevented. This analysis represents an important contribution in understanding the cost-effectiveness of population genomic screening by developing explicit models for seven genetic conditions and considering long-term impacts on life expectancy, patient disability, and health-care costs.

The results of the study are intriguing and provocative. If population genomic screening is so clearly cost-effective, should it be implemented in health-care systems? A critical look at the findings is warranted. A clear understanding of the analysis is challenging because of the number of conditions studied and the inherent intricacies of screening interventions. However, recognizing that the vast majority of clinical and patient benefit was derived from preventing breast, ovarian, and colon cancer can simplify this task. Specifically, of a screened population of 2.67 million young adults, the authors project that a combined 73,728 DALYs would be prevented (DALYs can be thought of as years of life lost). Of this total, $45 \%$ of DALYs were derived from preventing breast cancer, $21 \%$ from ovarian cancer, and $17 \%$ from colon cancer. The other modeled conditions contribute much less to the overall benefit of genomic screening due to their low population prevalence.

The results for HBOC screening on their own are somewhat surprising (AUD\$12,973/DALY preventedhighly cost-effective). While BRCA1/2 screening has been shown to be cost-effective in high-risk populations, its value in the general population is likely lower despite decreasing test costs. How did this analysis arrive at such optimistic findings? The authors are to be commended for providing detailed results and a methods appendix that enable closer evaluation. We were able to reproduce the number of breast cancer cases prevented by recreating the decision model in the study. However, we were not able to reproduce the benefits of preventing a case of breast cancer.

The results in Table 1 of Zhang et al. ${ }^{1}$ show 1278 cases of breast cancer would be prevented, and 617 deaths. Yet with 32,984 DALYs prevented, the model is estimating 53.5 DALYs per death prevented. In contrast, a prior study ${ }^{2}$ of HBOC population genomic screening estimated a benefit of approximately 9 quality-adjusted life-years (QALYs) (similar to DALY) per death from breast or ovarian cancer prevented, and recent work by our group has produced results that agree with this much lower estimate. ${ }^{3}$ The benefits for colon cancer prevention likewise appear to be higher than would be expected: 73.3 DALYs per case prevented and 121.8 DALYs per death prevented. Indeed, these estimates exceed human life expectancy and are thus difficult to understand, particularly considering that future events and life expectancy are discounted in economic evaluations.

It is possible that the overall benefits calculated in the model are being derived not by the $<1 \%$ of patients with a finding, but the remaining $99 \%$ without a finding. However, because of the potential for false positives, and the potential for individuals without findings to decrease adherence with standard recommended cancer screening, any potential benefits in this group (e.g., from the "value of knowing" their results) would likely be outweighed by potential harms. Another potential source of overall benefit is cascade screening in relatives of those with a positive finding, but Zhang and colleagues did not consider this in their analysis.

Cost-effective population genomic screening is one of the aspirational goals of precision medicine. Because of its complexity and uncertainty, understanding its potential cost-effectiveness will require multiple evaluations by independent investigators in an iterative fashion. The analysis by Zhang and colleagues has helped move the field forward by synthesizing the effects of screening for seven genetic conditions. The authors can deepen our understanding by providing a clearer explanation of how patient benefits were calculated, and by providing corrected estimates if needed.

\section{ACKNOWLEDGEMENTS}

This work was supported by the National Human Genome Research Institute (NHGRI 1 R01 HG009694-01).

\section{DISCLOSURE}

The authors declare no conflicts of interest.

Publisher's note: Springer Nature remains neutral with regard to jurisdictional claims in published maps and institutional affiliations.

David L. Veenstra, PharmD, $P h D$ (i) $^{1}$, Greg Guzauskas, $M S P H, P h D \mathbb{D}^{1}$, Josh Peterson, $M D, M P H^{2}$, Dina A. Hassen, $M P P^{3}$, Susan Snyder, PhD, $M B A^{3}$, Jing Hao, $P h D, M D^{3}$ and Marc Williams, $M D$ (i) $^{3}$

${ }^{1}$ Department of Pharmacy, University of Washington, Seattle, WA, USA; ${ }^{2}$ Departments of Biomedical Informatics and Medicine, Vanderbilt University, Nashville, TN, USA; ${ }^{3}$ Geisinger, Danville, PA, USA. Correspondence: David L. Veenstra (veenstra@uw.edu) 


\section{REFERENCES}

1. Zhang L, Bao Y, Riaz M, Tiller J, Liew D, Zhuang $X$, Amor DJ, Huq A, Petelin L, Nelson M, James PA, Winship I, McNeil JJ, Lacaze P. Population genomic screening of all young adults in a health-care system: a costeffectiveness analysis. Genet Med. 2019. https://doi.org/10.1038/ s41436-019-0457-6 [Epub ahead of print].

2. Manchanda R, Patel S, Gordeev VS, Antoniou AC, Smith S, Lee A, Hopper JL, Macinnis RJ, Turnbull C, Ramus SJ, Gayther SA, Pharoah PDP, Menon U, Jacobs I, Legood R. Cost-effectiveness of populationbased BRCA1, BRCA2, RAD51C, RAD51D, BRIP1, PALB2 mutation testing in unselected general population women. J Natl Cancer Inst. 2018;110:714-725.

3. Guzauskas GF, Spencer SJ, Veenstra DL. Timing and the costeffectiveness of population-wide genomic screening for breast and ovarian cancer. Paper presented at: American College of Medical Genetics Annual Meeting, Seattle, WA; 2019.

Advance online publication 15 July 2019. doi:10.1038/s41436-019-0580-4 